\title{
Antiretroviral medications disrupt microglial phagocytosis of $\beta$-amyloid and increase its production by neurons: Implications for HIV-associated neurocognitive disorders
}

Brian Giunta ${ }^{1,2^{*}}$, Jared Ehrhart ${ }^{3,4}$, Demian F Obregon ${ }^{3}$, Lucy Lam ${ }^{1}$, Lisa Le ${ }^{5}$, JingJi Jin ${ }^{1}$, Francisco Fernandez ${ }^{1,3}$, Jun $\operatorname{Tan}^{2,3}$ and R Douglas Shytle

\begin{abstract}
Up to $50 \%$ of long-term HIV infected patients, including those with systemically well-controlled infection, commonly experience memory problems and slowness, difficulties in concentration, planning, and multitasking. Deposition of $A \beta$ plaques is also a common pathological feature of HIV infection. However, it is not clear whether this accumulation is due to AD-like processes, HIV-associated immunosuppression, Tat protein-induced A $\beta$ elevations, and/or the effects of single highly active antiretroviral therapy (ART). Here we evaluated the effects of several ART medications (Zidovudine, Lamivudine, Indinavir, and Abacavir) alone and in combination on: 1) A $\beta_{1-40,42}$ generation in murine N2a cells transfected with the human "Swedish" mutant form of APP; 2) microglial phagocytosis of FITC$A \beta_{1-42}$ peptides in cultured murine N9 microglia. We report for the first time that these antiretroviral compounds (10 $\mu \mathrm{M})$ generally increase A $\beta$ generation ( 50-200\%) in SweAPP N2a cells and markedly inhibit microglial phagocytosis of FITC-A $\beta_{1-42}$ peptides in murine microglia. The most significant amyloidogenic effects were observed with combined ART ( $p<0.05)$; suggesting certain ART medications may have additive amyloidogenic effects when combined. As these antiretroviral compounds are capable of penetrating the blood brain barrier and reaching the concentrations employed in the in vitro studies, these findings raise the possibility that ART may play a casual role in the elevated $A \beta$ found in the brains of those infected with HIV. Therefore these compounds may consequently contribute to cognitive decline observed in HIV associated neurocognitive disorders (HAND).
\end{abstract}

Keywords: antiretrovirals, microglial cells, HIV, cognitive disorders

\section{Introduction}

Cognitive impairment occurs in a substantial (15-50\%) proportion of HIV-infected patients [1-3]. HIV-associated dementia (HAD) represents the most severe form [4]. With the introduction of antiretroviral therapy (ART), the incidence of HAD has dramatically decreased. In the past several years, patients-both longterm infected and treated-including those with systemically well-controlled infection, began to report milder memory problems and slowness, difficulties in

\footnotetext{
* Correspondence: bgiunta@health.usf.edu

${ }^{1}$ Neuroimmunology Laboratory, Department of Psychiatry and Neurosciences, College of Medicine, University of South Florida, Tampa, FL, 33612, USA

Full list of author information is available at the end of the article
}

concentration, planning, and multitasking; collectively termed HIV-associated neurocognitive disorders (HAND; [2]).

Although the pathological mechanism underlying HAND is unclear, an abundance of clinical and laboratory investigations suggest that HIV proteins, advanced age, and co-morbid neurodegenerative disease may interact in an additive or even synergistic manner resulting in the clinical presentation of this disorder $[5,6]$. This is concerning as there is an estimated 60,000 HIVinfected individuals over the age of 50 and 10,000 over the age of 65 . Furthermore, it has been predicted that $50 \%$ of prevalent acquired immunodeficiency syndrome (AIDS) cases in the United States will fall into this older age group by the year 2015 [7]. 
Interestingly, with such increased survival times imparted by ART, the prevalence of HAND is on the rise. At present, it is not clear whether this increased prevalence is due to the intrinsic risk of developing dementia with age or due to other direct or indirect factors of ART. In a recent clinical study, it was found that neurocognitive functioning significantly improved after immune competent, HIV-infected patients discontinued ART treatment. Moreover, this improvement continued in the patients remaining off ART over the nearly two year period of follow-up. Additionally, there was a lack of substantial neurocognitive improvement with resumption of ART [8].

There are several potential explanations underlying this clinically meaningful finding. One of these is that ART may lead to neurotoxicity [9] which is manifested in part by dysregulated neuronal amyloid precursor protein (APP) processing and concurrent deposition of amyloid beta $(A \beta)$ plaques in the brain.

While extracellular amyloid plaques are the primary amyloid pathology in Alzheimer's disease (AD), intraneuronal amyloid accumulation or perivascular diffuse amyloid depositions are more of a feature of HAND [3]. Strong evidence indicates this increased amyloid deposition in the brain of HIV-1-infected patients [10] as opposed to true full blown AD-like pathology. A correlation between the years of infection and amyloid deposition has also been shown [11]. Further, this amyloid deposition is most prevalent in the hippocampus and frontal lobe regions [12], and also observed in pyramidal neurons and along axonal tracks in HIV infected patients. Importantly, patients with HIV-associated encephalitis (HIVE) had higher levels of intraneuronal $A \beta$ immunoreactivity compared to HIV-1 patients without HIVE. In addition, intracellular deposition of $\mathrm{A} \beta$ correlated with age in the group of patients with HIVE [13]. Finally, HAND in older populations is at least partially linked to early signs of $\beta$-amyloidosis observed in $A D$, further demonstrating the importance of $A \beta$ deposition for the clinical outcome of HIV-1 infection. These studies raise questions regarding possible iatrogenic mechanisms involved in the pathogenesis of both HAND and/or AD as a co-morbid neurodegenerative [3,13].

In the present pilot study, we investigate the potential effects of various ART medications on neuronal A $\beta$ production and clearance by microglial phagocytosis in murine N2a cells transfected with the human "Swedish" mutant form of APP (SweAPP N2a cells) and N9 microglia cultures, respectively.

\section{Methods}

\section{Antiretrovirals}

The following reagents were obtained through the NIH AIDS Research and Reference Reagent Program,
Division of AIDS, NIAID, NIH: Zidovudine (AZT; Cat.\# 3485), Abacavir (Cat.\# 4680), Indinavir (Cat.\# 8145), and Lamivudine (3TC; Cat\# 8146).

\section{Neuronal A $\beta$ Production Assay}

SweAPP N2a cells were seeded at $1 \times 10^{5}$ cells/well $(\mathrm{n}=$ 2 for each condition) in 24-well tissue-culture plates containing $0.5 \mathrm{~mL}$ of complete medium (MEM medium supplemented with $10 \%$ fetal calf serum). Cells were differentiated prior to treatment with cAMP in $0.5 \mathrm{~mL}$ Neurobasal media for 4 hours. Following differentiation, these cells were untreated (control vehicle; PBS) or treated with ART medications both alone $(10 \mu \mathrm{M})$ and in combination $(10 \mu \mathrm{M})$ for 18 hours. $A \beta_{1-40,42}$ peptides were detected directly from the conditioned media and quantified in these samples using $\mathrm{A} \beta_{1-40,42}$ ELISA kits in accordance with the manufacturer's instructions.

\section{Microglial Phagocytosis Assay}

N9 microglia were seeded at $1 \times 10^{5}$ cells/well $(n=4$ for each condition) in 24-well tissue-culture plates containing $0.5 \mathrm{~mL}$ of complete medium (RPMI 1640 medium supplemented with $5 \%$ fetal calf serum). In the presence "aged" $\mathrm{A} \beta_{1-42}$ peptide conjugated with FITC (supplied by BioSource, used at $300 \mathrm{nM}$ dissolved in $\mathrm{dH}_{2} \mathrm{O}$ and pre-incubated for $24 \mathrm{~h}$ at $37^{\circ} \mathrm{C}$ ) microglia were co-treated with retroviral drugs both alone (10 $\mu \mathrm{M})$ and in combination $(10 \mu \mathrm{M})$ for $0,30,60,120$, and 180 minutes. For fluorometric analysis, in parallel treatments, the microglia were rinsed 3 times with complete medium after collection of cell supernatants and subjected to cell lysate preparation $[14,15]$. The total cellular protein of all groups was quantified and adjusted using the Bio-Rad protein assay. Extracellular and cell associated FITC-tagged $A \beta$ was quantified using an SPECTRAmax GEMINI microplate fluorometer (Molecular Devices Corp.) with an emission wavelength of $538 \mathrm{~nm}$ and an excitation wavelength of $485 \mathrm{~nm}$. In addition, in parallel 24-well tissue-culture plates, microglial cells were incubated at $4{ }^{\circ} \mathrm{C}$ with FITC-conjugated $\mathrm{A} \beta$ with or without the various antiretroviral treatments as controls for non-specifically incorporated A $\beta$. Microglial cells were rinsed 3 times in $A \beta$-free complete medium, and the media was exchanged with fresh $A \beta$-free complete medium for $10 \mathrm{~min}$ both to allow for removal of non-incorporated $A \beta$ and to promote concentration of the $A \beta$ into phagosomes. The relative mean fluorescence values for each sample at $37^{\circ} \mathrm{C}$ and $4^{\circ} \mathrm{C}$ at the indicated time points were determined by fluorometric analysis. Relative mean values were calculated as: (mean fluorescence value for each sample at $37^{\circ} \mathrm{C}$ - mean fluorescence value for each sample at $4^{\circ} \mathrm{C}$ ). In this manner, both extracellular and cell associated FITC-labeled $\mathrm{A} \beta$ were quantified. 


\section{Statistical Analysis}

All data were normally distributed; therefore, in instances of single mean comparisons, Levene's test for equality of variances followed by $t$-test for independent samples was used to assess significance. In instances of multiple mean comparisons, analysis of variance (ANOVA) was used, followed by post-hoc comparison using Bonferonni's method/correction. Alpha levels were set at 0.05 for all analyses. The statistical package for the social sciences release 10.0.5 (SPSS Inc., Chicago, IL, USA) was used for all data analysis.

\section{Results}

\section{ART medications increase $A \beta$ generation in cultured} SweAPP N2a cells

ART regimens are typically comprised of two major drug classes: protease inhibitors (PIs) and inhibitors of reverse transcriptase. The latter is subdivided into nucleoside reverse transcriptase inhibitors (NRTIs) and nonnucleoside reverse transcriptase inhibitors (nNRTIs). To examine the potential effects of a typical ART regimen on amyloidosis, we treated SweAPP N2a cells with $10 \mu \mathrm{M}$ concentrations of various antiretroviral compounds both alone and in combination for 18 hours. Following ELISA of the cultured media, we found that all compounds generally increased ( 50-200\%) A $\beta_{1-40,42}$ production (Figure 1). Interestingly, significant increases were observed when antiretroviral compounds were used in combination $(p<0.05$, Figure 1). Although we observe considerable increases in $\mathrm{A} \beta_{1-40,42}$ generation with all of the antiretroviral

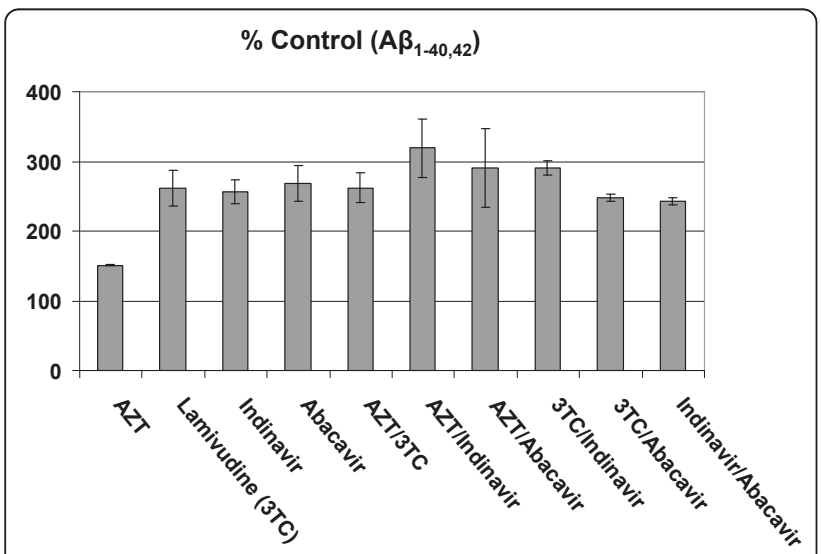

Figure 1 ART medications increase $A \beta$ generation in cultured neuronal cells. Total A $\beta_{1-40,42}$ peptides were analyzed in conditioned media from SweAPP N2a cells by ELISA ( $n=2$ for each condition). Data are represented as a mean \pm SEM percentage of A $\beta_{1-40,42}$ peptides secreted 18 hours after antiretroviral treatment relative to control (untreated). One-way ANOVA followed by post hoc comparison revealed significant increases in $A \beta_{1-40,42}$ production following 3TC/Indinavir, 3TC/Abacavir, and Indinavir/ Abacavir treatements (concentration of $10 \mu \mathrm{M}$ for each individual compound; $p<0.05$ ). compounds, it appears that combinations of the NRTIs Lamivudine (3TC) and/or Abacavir, with, the PI Indinavir, exert the most significant amyloidogenic effects. Notably, the NRTI Zidovudine (AZT) conferred significantly less $A \beta_{1-40,42}$ production as compared to the antiretroviral combinations mentioned above $(p<0.05$; Figure 1$)$. Taken together, the data suggests that iatrogenic mechanisms may in fact contribute to HAND and AD-like pathology in HIVinfected individuals.

\section{ART medications inhibit microglial phagocytosis of $A \beta_{1-42}$ peptides}

To determine whether ART could affect microglial clearance of $\mathrm{A} \beta$ and further promote amyloidosis, we performed a phagocytosis assay with N9 cells in the presence of antiretroviral compounds both alone and in combination. Following detection of FITC-tagged A $\beta_{1-42}$ in extracellular and cell associated fractions, we again found that all compounds generally inhibited microglial phagocytosis/clearance (Figure 2). All antiretroviral compounds significantly inhibited microglial phagocytosis of $A \beta_{1-42}$ peptides as determined by high levels of peptide remaining in the cultured media (extracellular) $(p<0.05$, Figure $2 \mathrm{a})$. In addition, a majority of the compounds tested also significantly reduced levels of phagosomal (cell associated) $\mathrm{A} \beta_{1-42}(p<0.05$, Figure $2 \mathrm{~b})$. Also, when comparing cell associated $A \beta_{1-42}$ levels of the Indinavir/Abacavir combination to levels of these compound alone, the differences suggest the combination of this PI and NRTI is additive in nature $(p<0.05$; Figure 2b). Importantly, when comparing the levels of extracellular $A \beta_{1-42}$ (Figure $2 a$ ) to that of cell associated (Figure $2 \mathrm{~b}$ ) we can see that the phagocytosis/clearance profiles are relatively congruent for each treatment condition. That is to say, when a given treatment maintains high levels of extracellular $A \beta_{1-42}$, the corresponding cell associated levels are relatively low. Not only does this apparent relationship between extracellular and cell associated $A \beta_{1-42}$ levels confirm the accuracy of the assay, but also furthers the overall significance of the inhibition of microglial phagocytosis by the antiretrovirals.

\section{Discussion}

Therapy with at least three ART medications has been standard treatment for HIV infected patients for since approximately 1995 [16]. Indeed combination ART has dramatically reduced medical morbidity and mortality with HIV infection, but high rates of HAND continue to be reported [17]. Heaton and colleagues sought to determine neurocognitive impairment in large groups of HIV + and HIV - participants from the pre-combination ART era (1988-1995; $\mathrm{N}=857$ ) and combination ART era (2000-2007; $N$ =937). The rates of impairment increased with successive disease stages in both eras: 


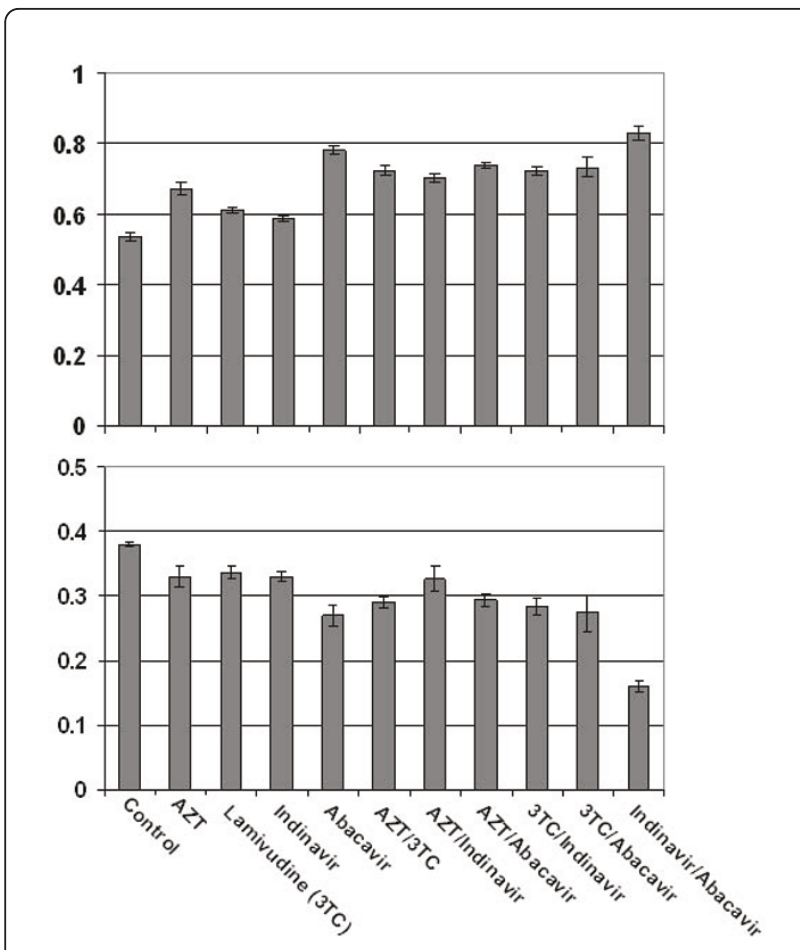

Figure 2 ART medications inhibit microglial phagocytosis of $\mathbf{A} \boldsymbol{\beta}_{1-42}$ peptides. N9 microglia were treated with "aged" FITCtagged $A \beta_{1-42}(300 \mathrm{nM})$ in complete medium for $120 \mathrm{~min}$ in the presence of $10 \mu \mathrm{M}$ concentrations of antiretrovirals. Total FITC-A $\beta_{1-42}$ peptides were analyzed by fluorescence from conditioned media (extracellular; top) and cell lysates (phagosomal/cell associated; bottom). Data are represented as the relative mean \pm SEM

fluorescence ( $n=4$ for each condition presented). When measuring extracellular FITC-tagged A ${ }_{1-42}$, one-way ANOVA followed by post hoc comparison showed significantly higher levels following all antiretroviral treatments, as compared to control $(p<0.05)$. When measuring cell associated FITC-tagged A ${ }_{1-42}$, one-way ANOVA followed by post hoc comparison showed significantly lower levels following Indinavir, Abacavir, AZT/3TC, AZT/Abacavir, 3TC/Indinavir, and Indinavir/Abacavir, as compared to control $(p<0.05)$.

$25 \%, 42 \%$, and $52 \%$ in pre-ART era and $36 \%, 40 \%$, and $45 \%$ in combination ART era. In the medically asymptomatic stage, neurocognitive impairment was significantly more common in the ART era; indicating a possible iatrogenic mechanism whereby ART is salutary in the periphery, but possibly in some way deleterious in the CNS [17]. Furthermore, the pattern of neurocognitive impairment also differed. Patients from the pre-ART era had more impairment in motor skills, cognitive speed, and verbal fluency. On the other hand, ART era patients suffered more memory (learning) and executive function deficits. Importantly, this study showed high rates of mild neurocognitive impairment persist at all stages of HIV infection, despite adequate viral suppression and immune reconstitution with combination ART [17].
In this context, we sought to identify the risk of adverse neuropathological side effects from various ART regimens in vitro. Here, we elucidate a potential mechanism whereby antiretroviral compounds may have neurotoxic effects, both alone and in combination. This may contribute to the neurological complications that are associated with advanced HIV infection and/or long-term ART seen clinically. Our study shows antiretroviral compounds may effectively increase $\mathrm{A} \beta$ generation while possessing the capability to inhibit its clearance by preventing microglial phagocytosis. By affecting both amyloidogenic fronts (generation and clearance), antiretroviral treatment may substantially enhance $A \beta$ aggregation and deposition, which itself is neurotoxic.

We find the most significant amyloidogenic effects when the antiretroviral compounds 3TC, Indinavir, and Abacavir are used in combination (Figure 1). Also, we observe that particular antiretrovirals, Indinavir and Abacavir, may have detrimental additive effects on $A \beta$ microglial clearance (Figure 2). Accordingly, it is likely that certain 3 drug regimens may present an even greater risk of neurological complications.

Though higher CNS-penetrating regimens have been associated with neurocognitive improvement, recent research demonstrates ART might also impart neurotoxic effects; adversely affecting cognition. Indeed in a recent clinical study discontinuation of ART in experienced subjects improved neurocognition and those results were not attributed to practice effects. Furthermore, subjects reinitiated on ART did not experience cognitive gains. Therefore, ART neurotoxicity might explain the unexpected results of this clinical study gains [8]. Past reports have indicated the deleterious effects of ART on the brain were at least in part caused by damage to peripheral neural tissues (for review see [9]). NRTIs have been shown to induce toxicity in peripheral tissues by altering mitochondrial function, and PIs were shown to damage proteosome function $[12,18]$. Also, subjects on didanosine and stavudine regimens had decreased $\mathrm{N}$-acetylaspartate (NAA) concentrations in frontal white matter, a sign of neurotoxicity which positively correlated with treatment duration [19].

Recently, studies have addressed the influence of five NRTIs (2' 3'-dideoxyinosine, zidovudine, emtricitabine, and tenofovir), one NNRTI (efavirenz), and two PIs (ritonavir, atazanavir sulfate) on neuronal integrity and function. All of the antiretroviral medications tested except for 2' 3'-dideoxyinosine reduced mitochondrial membrane potential. Furthermore, several antiretroviral medications destabilized neuronal intracellular calcium homeostasis, showing a reduced acute response to glutamate [20]. The ability of certain antiretroviral medications and combinations thereof to dysregulate neuronal calcium homeostasis and affect the mitochondrial 
membrane potential both promote the deposition of $A \beta$ plaques and increased amyloidogenic processing of APP (for review see [21]). Furthermore, neurons treated with antiretroviral medications exhibited dendritic beading and pruning correlated over a range of doses, which has been linked to cognitive dysfunction [22].

In our experiments, the median toxic doses for several ARVs were well within the therapeutic concentration range in plasma of HIV-infected patients, and a few showed some signs of damage in the range of CSF concentrations. These initial observations highlight potential adverse effects of high concentrations of antiretroviral medications in the CNS and indicate that there may be some negative tradeoffs to traditionally delivering "therapeutic concentrations" of these compounds to the CNS.

As previous pharmacokinetic studies have confirmed the moderate to high oral bioavailabilities and low to moderate plasma protein binding properties of 3TC, Indinavir, and Abacavir, it is feasible that all of these antiretroviral compounds can reach systemic peak concentrations $>100 \mathrm{uM}$ following normal dosing regimens. Needless to say, only a fraction of these concentrations needs to be distributed in the brain to mediate the amyloidogenic effects we observed in our in vitro studies, which employed 10uM concentrations. Furthermore, ART effects in vivo are likely to occur over long-term exposures. Chronic, low dose, in vivo effects of any reagent are often very appropriately modeled in vitro, by proportionately higher doses of that same reagent, over more acute time frames [23]. For these reasons we used 10uM ART dose for these experiments.

This brings us to a potential dilemma in ART concerning an important parameter, blood brain barrier (BBB) permeability. On one hand 3TC, Abacavir, and Indinavir have been reported to be moderately $\mathrm{BBB}$ permeable and consequently may be free to promote amyloidosis. On the other hand, these antiretroviral compounds may also be more capable of reducing HIV load in the brain, which may be essential to avoid general HIV encephalopathy. Furthermore, Liu and colleagues [24] demonstrated that the HIV-1 Tat protein competitively inhibits the LRP receptor, resulting in an inhibition of $A \beta$ clearance. Therefore, by minimizing HIV replication and associated Tat protein expression in the brain, $\mathrm{BBB}$ permeable antiretroviral compounds may also prevent amyloidosis. In light of this complex situation, switching from an ART regimen that is associated with both amyloidosis and the potentially related lipodystrophy to one without these adverse effects may be the best course of clinical action to reduce the risk of neurological complications. On the other hand, consistent association of neurocognitive impairment with nadir CD4 across pre-and post-ART eras suggests that earlier treatment to prevent severe immunosuppression may also help prevent HAND. Clinical trials targeting HAND prevention should specifically examine timing of ART initiation [2].

Future studies are also warranted to investigate the dose-response effects of newer BBB permeable antiretroviral drugs including fusion inhibitors, alone and in combination, on microglial CNS A $\beta$ levels in an in vivo murine model. Moreover, future studies will be required to determine the effect of antiretrovirals on APP proteolysis and microglial $A \beta$ proteases including neprilysin, insulin-degrading enzyme, and endothelin-converting enzymes 1 and 2, as ART modulated proteolytic activity could affect the development of HAND and amyloidosis. This study was also limited to the effect of ART of microglia and neurons separately without other mitigating factors. In the future this work will need to be replicated in vivo in mouse models to not only account for astrocytes, but the entire brain milieu. Nevertheless, it is clear from the most recent update by the World Health Organization in 2010 that the cost of rapid ART scaleup is significant in terms of side-effects [16]. There is a need from patients and health-care providers to phase in less toxic ART regiments while maintaining simplified fixed-dose combinations.

\section{Acknowledgements}

This work was in part supported by Johnnie B. Byrd, Sr. Alzheimer's Center \& Research Institute (DS and JT) and the NIH/NIA/NIMH (JT and BG). Antiretroviral medications were provided by AIDS Research and Reference Reagent Program.

\section{Author details}

${ }^{1}$ Neuroimmunology Laboratory, Department of Psychiatry and Neurosciences, College of Medicine, University of South Florida, Tampa, FL, 33612, USA. ${ }^{2}$ Department of Molecular Medicine, Department of Psychiatry and Neurosciences, College of Medicine, University of South Florida, Tampa FL, 33612, USA. ${ }^{3}$ Silver Child Development Center, Department of Psychiatry and Neurosciences, College of Medicine, University of South Florida, Tampa, FL, 33612, USA. ${ }^{4}$ Center Excellence in Aging and Brain Repair, Department of Neurosurgery, College of Medicine, University of South Florida, Tampa, FL, 33612, USA. ${ }^{5}$ Department of Pharmacology and Physiology, College of Medicine, University of South Florida, Tampa, FL 33612, USA.

\section{Authors' contributions}

BG and RS drafted the manuscript. JE and DO carried out the immunoassays. JJ, LL, and LL performed statistical analysis. FF conceived of the study. JT participated in its design and coordination. All authors read and approved the final manuscript.

\section{Competing interests}

The authors declare that they have no competing interests.

Received: 8 March 2011 Accepted: 7 June 2011 Published: 7 June 2011

\section{References}

1. Goodkin K, Fletcher MA, Cohen N: Clinical aspects of psychoneuroimmunology. Lancet 1995, 345(8943):183-4

2. Schouten J, et al: HIV-1 infection and cognitive impairment in the CART era: a review. AIDS 2011, 25(5):561-75.

3. Xu J, lkezU T: The comorbidity of HIV-associated neurocognitive disorders and Alzheimer's disease: a foreseeable medical challenge in post-HAART era. J Neuroimmune Pharmacol 2009, 4(2):200-12. 
4. Shapshak P, et al: Elevated expression of IFN-gamma in the HIV-1 infected brain. Front Biosci 2004, 9:1073-81.

5. Ghafouri M, et al: HIV-1 associated dementia: symptoms and causes. Retrovirology 2006, 3:28.

6. Gonzalez-Scarano F, Martin-Garcia J: The neuropathogenesis of AIDS. Nat Rev Immunol 2005, 5(1):69-81.

7. Aging Hearing: HIV over fifty, exploring the new threat. 2005, (Senate Committee on Aging).

8. Robertson KR, et al: Neurocognitive effects of treatment interruption in stable HIV-positive patients in an observational cohort. Neurology 2010, 74(16):1260-6.

9. Liner KJ, Ro MJ, Robertson KR: HIV, antiretroviral therapies, and the brain. Curr HIV/AIDS Rep 2010, 7(2):85-91.

10. Esiri MM, Biddolph SC, Morris CS: Prevalence of Alzheimer plaques in AIDS. J Neurol Neurosurg Psychiatry 1998, 65(1):29-33.

11. Rempel HC, Pulliam L: HIV-1 Tat inhibits neprilysin and elevates amyloid beta. AIDS 2005, 19(2):127-35

12. Brew BJ, et al: Neurodegeneration and ageing in the HAART era. $J$ Neuroimmune Pharmacol 2009, 4(2):163-74

13. Achim CL, et al: Increased accumulation of intraneuronal amyloid beta in HIV-infected patients. J Neuroimmune Pharmacol 2009, 4(2):190-9.

14. Giunta B, et al: HIV-1 TAT inhibits microglial phagocytosis of Abeta peptide. Int I Clin Exp Pathol 2008, 1(3):260-75.

15. Townsend $\mathrm{KP}$, et al: $\mathrm{CD} 40$ signaling regulates innate and adaptive activation of microglia in response to amyloid beta-peptide. Eur Immunol 2005, 35(3):901-10.

16. Antiretroviral therapy for HIV infection in adults and adolescents: recommendations for a public health approach. 2010, (World Health Organization).

17. Heaton RK, et al: HIV-associated neurocognitive disorders persist in the era of potent antiretroviral therapy: CHARTER Study. Neurology 2010, 75(23):2087-96.

18. Cysique $L A$, Brew $B J$ : Neuropsychological functioning and antiretroviral treatment in HIV/AIDS: a review. Neuropsychol Rev 2009, 19(2):169-85.

19. Schweinsburg $B C$, et al: Brain mitochondrial injury in human immunodeficiency virus-seropositive (HIV+) individuals taking nucleoside reverse transcriptase inhibitors. J Neurovirol 2005, 11(4):356-64.

20. Dou $\mathrm{H}$, et al: Macrophage delivery of nanoformulated antiretroviral drug to the brain in a murine model of neuroAIDS. I Immunol 2009, 183(1):661-9.

21. Readnower RD, Sauerbeck AD, Sullivan PG: Mitochondria, Amyloid beta, and Alzheimer's Disease. Int J Alzheimers Dis 2011, 2011:104545.

22. Ellis $\mathrm{R}$, Langford D, Masliah E: HIV and antiretroviral therapy in the brain: neuronal injury and repair. Nat Rev Neurosci 2007, 8(1):33-44

23. Kiebala $M$, et al: Nuclear factor-kappa B family member RelB inhibits human immunodeficiency virus-1 Tat-induced tumor necrosis factoralpha production. PLoS One 2010, 5(7):e11875.

24. Liu Y, et al: Uptake of HIV-1 tat protein mediated by low-density lipoprotein receptor-related protein disrupts the neuronal metabolic balance of the receptor ligands. Nat Med 2000, 6(12):1380-7.

doi:10.1186/1756-6606-4-23

Cite this article as: Giunta et al: Antiretroviral medications disrupt microglial phagocytosis of $\beta$-amyloid and increase its production by neurons: Implications for HIV-associated neurocognitive disorders.

Molecular Brain 2011 4:23.

\section{Submit your next manuscript to BioMed Central and take full advantage of:}

- Convenient online submission

- Thorough peer review

- No space constraints or color figure charges

- Immediate publication on acceptance

- Inclusion in PubMed, CAS, Scopus and Google Scholar

- Research which is freely available for redistribution

Submit your manuscript at www.biomedcentral.com/submit 